\title{
Mineral Oil Scabies Preparation From Under the Fingernail
}

\author{
Kendahl Oberdorfer, BS; Meena Moossavi, MD, MPH
}

The microscopic mite Sarcoptes scabiei can burrow into the epidermis of human skin and cause scabies. Obtaining scabies mites from the skin for diagnosis can be challenging. We describe a method for extracting the scabies mite using the tip of a disposable blunt-tip hyfrecator electrode to gently scrape under the fingernail bed. We find that this is a quick and efficient method of obtaining a scabies mite for rapid diagnosis.

Cutis. 2021;108:228-229.

\section{Practice Gap}

The Sarcoptes scabiei mite is a microscopic organism that causes scabies in the human host. The scabies mite is highly transmissible, making scabies a common disease in heavily populated areas. The mite survives by burrowing into the epidermis, where it feeds, lays eggs, and defecates. ${ }^{1}$

The rash in the host represents an allergic reaction to the body of the scabies mite, producing symptoms such as intense itching, rash, and erosions of the skin. The scabies rash tends to occur in warm and occluded areas of the body such as the hands, axillae, groin, buttocks, and feet. ${ }^{1,2}$

Delaying treatment of scabies can be hazardous because of the risk of rapid spread from one person to another. This rapid spread can be debilitating in specific populations, such as the immunocompromised, elderly, and disabled.

Mineral oil preparation is the classic method used to identify scabies (Figure 1). This method relies on obtaining mites by applying mineral oil to the skin and using a 15-mm blade to scrape off layers of the affected skin. The scraped material is spread onto a microscope slide with mineral oil, a coverslip is applied, and the specimen is analyzed by direct microscopy. This method proves only as effective as knowing where the few mites are located.
At any time, only 10 to 12 mites live on a human host. $^{3}$ Therefore, it can be challenging to obtain a mite for diagnosis because the location of the skin mites may be unknown. Dermoscopy can be used to locate burrows and other signs of $S$ scabiei. With a dermatoscope, the scabies mite can be identified by the so-called delta-wing jet sign. ${ }^{4}$

However, dermoscopy is not always successful because extensive hemorrhagic crusting and erosions of the skin secondary to constant scratching can obscure the appearance of burrows and mites. Because patients are constantly scratching areas of irritation, it is possible that $S$ scabiei can be located under the fingernail of the dominant hand.

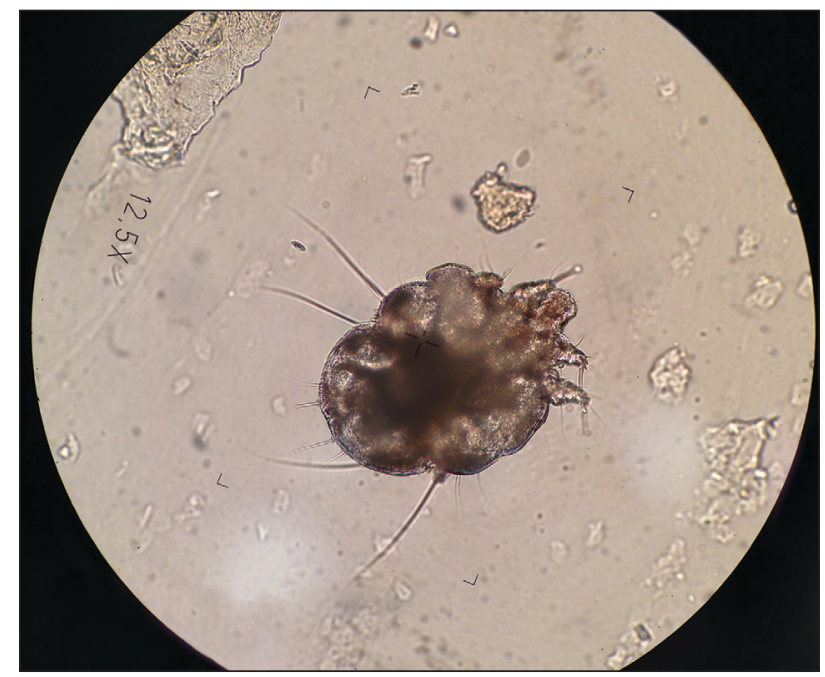

FIGURE 1. Sarcoptes scabiei mineral oil preparation (original magnification $\times 400$ ). Microphotograph courtesy of Steven Daveluy, MD (Detroit, Michigan).

Ms. Oberdorfer is from Wayne State University School of Medicine, Detroit, Michigan. Dr. Moossavi is from the Department of Dermatology, John D. Dingell VA Medical Center, Detroit.

The authors report no conflict of interest.

Correspondence: Meena Moossavi, MD, MPH, 4646 John R. St, Detroit, Ml 48201 (moossav@wayne.edu).

doi:10.12788/cutis.0360 


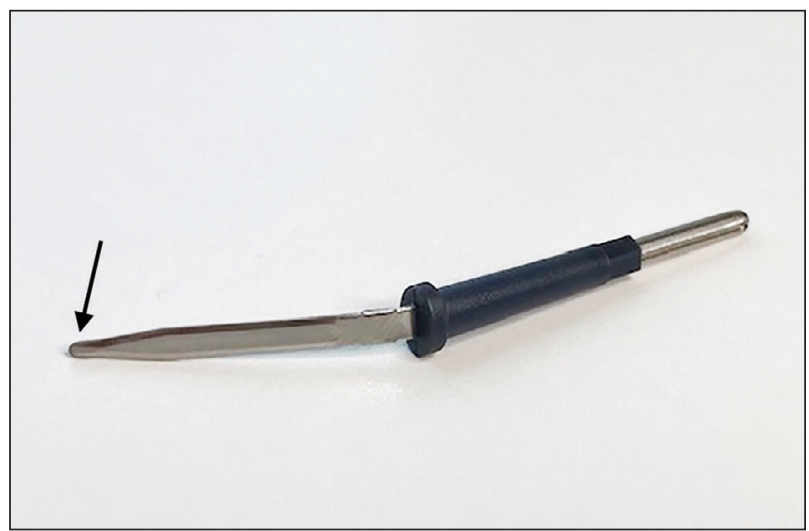

FIGURE 2. A disposable hyfrecator tip (black arrow).

\section{The Technique}

To address this practice gap, a mineral oil scabies preparation can be performed by scraping under the fingernail plate at the level of the hyponychium. Mites might accumulate underneath the fingernails of the dominant hand when patients scratch the area of the skin where $S$ scabiei mites are burrowing and reproducing.

A convenient and painless way to obtain a mineral oil scabies preparation from under the fingernail is to use the tip of a disposable hyfrecator, readily available in most dermatology practices for use in electrosurgery (Figure 2). Using the blunt end of the hyfrecator tip for the mineral oil preparation would be done without attachment to the full apparatus.

The hyponychium of the fingernail is prepared with mineral oil, which aids in collecting and suspending the material obtained from under the nail plate. Using the blunt end of the hyfrecator tip, material from underneath the fingernail is removed using a gentle sweeping motion (Figure 3). The specimen is then analyzed under the microscope similar to a routine mineral oil scabies preparation. This method can be utilized by health care providers for easy and painless diagnosis of scabies.

\section{Practice Implications}

Use of a blunt hyfrecator tip to extract $S$ scabiei from underneath the fingernail plate can be used for efficient diagnosis of scabies. This technique can be implemented

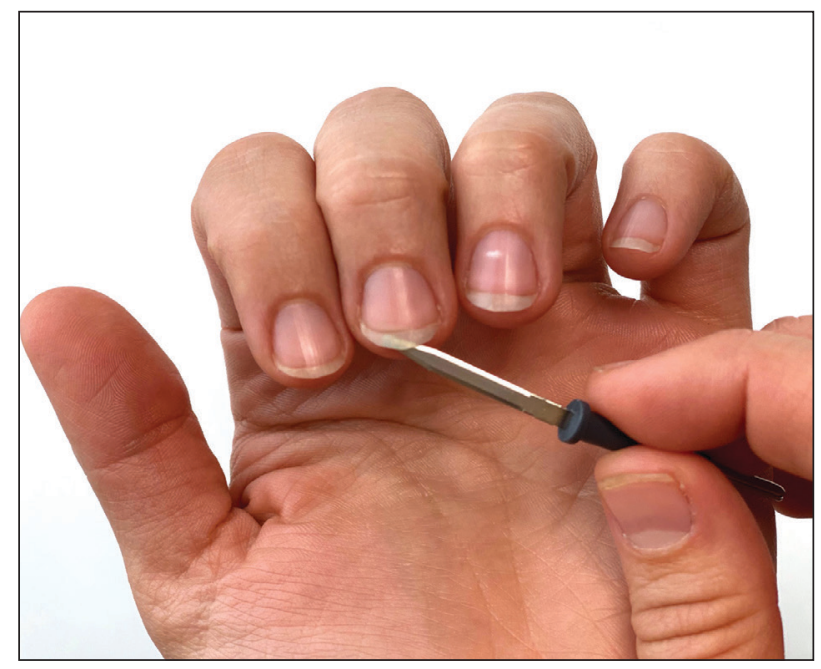

FIGURE 3. The blunt tip of the hyfrecator electrode is used to sweep under the fingernail to painlessly obtain Sarcoptes scabiei to diagnose scabies

in any clinic where blunt-tip hyfrecator electrodes are available. Using a gentle sweeping motion, the blunt-tip hyfrecator allows the provider to extract material from under the fingernail for diagnosis. The material obtained is used to prepare a mineral oil scabies preparation for direct microscopic analysis.

This technique can diagnose scabies efficiently, and treatment can be initiated promptly. Use of a disposable blunt-tip hyfrecator for scabies extraction is a novel technique that can be added to the armamentarium of tools to diagnose scabies, which includes traditional mineral oil preparation and dermoscopy.

\section{REFERENCES}

1. Banerji A; Canadian Paediatric Society, First Nations, Inuit and Métis Health Committee. Scabies. Paediatr Child Health. 2015;20:395-402. doi:10.1093/pch/20.7.395

2. Johnston G, Sladden M. Scabies: diagnosis and treatment. BMJ. 2005;331:619-622. doi:10.1136/bmj.331.7517.619

3. Mellanby K. The development of symptoms, parasitic infection and immunity in human scabies. Parasitology. 1944;35:197-206. doi:10.1017/S0031182000021612

4. Fox G. Diagnosis of scabies by dermoscopy [published online February 2, 2009]. BMJ Case Rep. 2009;2009:bcr06.2008.0279. doi:10.1136/bcr.06.2008.0279 